\title{
A periplasmic arsenite-binding protein involved in regulating arsenite oxidation
}

\author{
Authors: Guanghui Liu, Mengyao Liu, Eun-Hae Kim, \\ Walid S. Maaty, Brian Bothner, Benfang Lei, \\ Christopher Rensing, Gejiao Wang, and \\ Timothy R. McDermott
}

This is the peer reviewed version of the following article: [Liu, Guanghui, Mengyao Liu, Eun-Hae Kim, Walid S. Maaty, Brian Bothner, Benfang Lei, Christopher Rensing, Gejiao Wang, and Timothy R. McDermott. "A Periplasmic Arsenite-Binding Protein Involved in Regulating Arsenite Oxidation." Environmental Microbiology 14, no. 7 (December 19, 2011): 1624-1634], which has been published in final form at http://dx.doi.org/10.1111/j.1462-2920.2011.02672.x. This article may be used for non-commercial purposes in accordance with Wiley Terms and Conditions for Self-Archiving.

Liu, Guanghui, Mengyao Liu, Eun-Hae Kim, Walid S. Maaty, Brian Bothner, Benfang Lei, Christopher Rensing, Gejiao Wang, and Timothy R. McDermott. "A Periplasmic Arsenite-Binding Protein Involved in Regulating Arsenite Oxidation.” Environmental Microbiology 14, no. 7 (December 19, 2011): 1624-1634. doi:10.1111/j.1462-2920.2011.02672.x.

Made available through Montana State University's $\underline{\text { ScholarWorks }}$ scholarworks.montana.edu 


\title{
A periplasmic arsenite-binding protein involved in regulating arsenite oxidation
}

\author{
Guanghui Liu, ${ }^{1,2}$ Mengyao Liu, ${ }^{3}$ Eun-Hae Kim, ${ }^{5}$ Walid S. Maaty, ${ }^{4}$ Brian Bothner, ${ }^{4}$ Benfang Lei, ${ }^{3}$ Christopher \\ Rensing, ${ }^{5}$ Gejiao Wang ${ }^{1 \star *}$ and \\ Timothy R. McDermott ${ }^{2 *}$ \\ ${ }^{1}$ State Key Laboratory of Agricultural Microbiology, College of Life Science and Technology, Huazhong Agricultural \\ University, Wuhan 430070, China. Departments of ${ }^{2}$ Land Resources and Environmental Sciences, ${ }^{3}$ Immunology and \\ Infectious Disease, and ${ }_{4}^{4}$ Chemistry and Biochemistry, Montana State University, Bozeman, MT 59717, USA. \\ ${ }^{5}$ Department of Soil, Water, and Environmental Science, The University of Arizona, Tucson, AZ 85721, USA.
}

\begin{abstract}
Summary
Arsenic (As) is the most common toxic element in the environment, ranking first on the Superfund List of Hazardous Substances. Microbial redox transforma-tions are the principal drivers of As chemical specia-tion, which in turn dictates As mobility and toxicity. Consequently, in order to manage or remediate envi-ronmental As, land managers need to understand how and why microorganisms react to As. Studies have demonstrated a two-component signal trans-duction system comprised of AioS (sensor kinase) and AioR (response regulator) is involved in regulat-ing microbial AsIll oxidation, with the AsIll oxidase structural genes aio $B$ and aioA being upregulated by AsIII. However, it is not known whether AsIII is first detected directly by AioS or by an intermediate. Herein we demonstrate the essential role of a peri-plasmic Aslll-binding protein encoded by aioX, which is upregulated by AsIII. An DaioX mutant is defective for upregulation of the aioBA genes and conse-quently AsIll oxidation. Purified AioX expressed without its TAT-type signal peptide behaves as a monomer (MW $32 \mathrm{kDa}$ ), and Western blots show AioX to be exclusively associated with the cytoplasmic membrane. AioX binds AsIll with a KD of $2.4 \mathrm{mM}$ AsIll; however, mutating a conserved Cys108 to either alanine or serine resulted in lack of AsIII binding, lack of aioBA induction, and correlated with a negative AsIII oxidation phenotype. The discovery and charac-terization of AioX illustrates a novel Aslll sensing mechanism that appears to be used in a range of bacteria and also provides one of the first examples of a bacterial signal anchor protein.
\end{abstract}

\section{Introduction}

Transport and bioavailability of arsenic (As) in the envi-ronment is dependent on chemical speciation; hence, the abiotic and biotic processes that regulate arsenite (AsIII) oxidation and arsenate (AsV) reduction have important implications for watershed quality management in As-impacted environments. The various abiotic and biotic factors that control As fate and transport are not mutually exclusive; however, it is now understood that microbial As redox transformations are an important (if not the princi-pal) force controlling As speciation in most environments (Cullen and Reimer, 1989; Pontius et al., 1994; Inskeep et al., 2001; Oremland and Stolz, 2005; Stolz et al., 2006). Thus, in order to better understand microbe-As interac-tions in nature and to more effectively strategize bioremediation efforts, it is critical that there be a more formal and foundational understanding of how microbes sense and react to As.

At present, a fairly detailed model explaining the genet-ics, regulation and function of detoxification-based As $V$ reduction is in place. At the minimum, this involves pro-teins encoded by arsRBC: ArsR is a repressor controlling the expression of arsRBC, ArsB extrudes AsIll from the cell, and ArsC is an AsV reductase that converts AsV to Aslll, which is the substrate for ArsB (Bhattacharjee and Rosen, 2007). In addition, AsV reductase enzymes involved in anaerobic AsV respiration have been charac-terized from three organisms (Kraft and Macy, 1998; Afkar et al., 2003; Saltikov and Newman, 2003; Malasarn et al., 2007) and the encoding genes (arrAB) have been char-acterized (Saltikov and Newman, 2003).

Studies on the genetics and physiology of Aslll oxida-tion are at an initial stage. Early accomplishments include the characterization of one of the two identified AsIll oxi-dases (Phillips and Taylor, 1976; Anderson et al., 1992; Ellis et al., 2001). The genes coding for AsIll oxidase have been cloned (Muller et al., 2003; Santini and vanden 
Hoven, 2004), and more recently a phylogenetically distinct AsIll oxidase from Alkalilimnicola ehrlichii was cloned and characterized (Zargar et al., 2010). Note that in this report we are installing modified gene symbol nomenclature resulting from recent international discussions designed to unify the arsenite oxidase literature with respect to gene symbols and to eliminate confusion with other proteins. To that end, aox/aro/aso are all now designated aio (arenite oxidase), and the arsenite oxidase large subunit is designated as $\mathrm{A}$ and the small subunit as $\mathrm{B}$, e.g. aoxAB is now aioBA (see Lett et al., 2011).

Our previous efforts with Agrobacterium tumefaciens identified a two-component signal transduction pair, aioSR (previously aoxSR), as being essential to AsIll oxidation (Kashyap et al., 2006a). In addition, Kashyap and colleagues (2006b) found that a molybdate transporter and a $\mathrm{Na}^{+} / \mathrm{H}^{+}$antiporter are also essential for Aslll oxidation. Later, Koechler and colleagues (2010) using a similar transposon mutation approach also identified the aioSR two-component pair and molybdate transporter as being essential for AsIll oxidation, and in addition provided evidence that RpoN (alternative sigma factor, $\sigma^{54}$ ) and DnaJ (heat shock protein $\mathrm{J}$ ) are also required for this process. The experiments summarized in the current study take the next step in characterizing important regulatory elements that control bacterial AsIll oxidation. Specifically, we describe a gene and its encoded protein that is essen- tial for the upregulation of aio $A B$ and for AsIll oxidation. We present the initial characterization of the encoded protein, which behaves in a manner consistent with it being a periplasmic Aslll-binding protein.

\section{Results \\ Identification and expression analysis of aioX}

All mutants, plasmids and genetic constructs generated for this study are shown in Table 1. Previous work identified an open reading frame upstream of the arsenite oxidase regulatory locus in $A$. tumefaciens strain $5 \mathrm{~A}$ (Kashyap et al., 2006a). To understand its potential role in Aslll oxidation, the full coding sequence (921 bp) was determined by TAIL-PCR and sequencing, and is referred to as aio $X$ as it is a homologue of the aio $X$ gene referred to as aox X by Cai and colleagues (2009) and encodes a 306-amino-acid protein that shares significant identity and similarity with a number of variously annotated solutebinding proteins and can be found associated with aio genes in a range of Aslll oxidizing microbes (Figs S1 and S2). Because genes involved in Aslll oxidation are typically induced by AsIII, aioX expression was examined by quantitative reverse transcriptase (qRT)-PCR. Levels of aioX transcript in early log-phase cells in the presence of $100 \mu \mathrm{M}$ Aslll were $3615 \pm 411$ copies per nanogram of

Table 1. Bacterial strains and plasmids used in this study.

\begin{tabular}{|c|c|c|}
\hline Strain/plasmid & Relevant properties or derivation & Source or reference \\
\hline \multicolumn{3}{|l|}{ Strains } \\
\hline \multicolumn{3}{|l|}{ Agrobacterium tumefaciens } \\
\hline $5 \mathrm{~A}$ & Wild type, soil isolate, As(III) oxidizing & Macur et al. (2004) \\
\hline M53 & aio $X$ deletion mutant & This study \\
\hline \multicolumn{3}{|l|}{ Escherichia coli } \\
\hline $\mathrm{DH} 5 \alpha$ & supE44 lacU169(ب80lacZM15) hRDR17 recA1 endA1 gyrA96 thi-1 relA1 & Hanahan (1983) \\
\hline HB101 & supE44 hsdS20 $\left(\mathrm{r}_{\mathrm{B}}^{-} \mathrm{m}_{\mathrm{B}}^{-}\right)$recA13 ara-14 proA2 lacY1 galK2 rpsL20 xyl-5 metl-1 & $\begin{array}{l}\text { Boyer and Roulland-Dussoix } \\
\text { (1969) }\end{array}$ \\
\hline JM110 & $\mathrm{dam}$ and $\mathrm{dcm}$ deficient, cloning and expression host & Stratagene \\
\hline TOP10 & High-competency cloning host & Invitrogen \\
\hline BL21StarTM(DE3)pLysS & $\mathrm{F}^{-}$ompT hsdS ${ }_{B}\left(\mathrm{r}_{\mathrm{B}^{-}} \mathrm{m}_{\mathrm{B}^{-}}\right) \mathrm{gal} d \mathrm{dcm}$ me131 (DE3) pLys $\left(\mathrm{Cam}^{\mathrm{R}}\right)$ & Invitrogen \\
\hline \multicolumn{3}{|l|}{ Plasmids } \\
\hline pGEM-T & PCR TA cloning vector; $\mathrm{Amp}^{\mathrm{R}}$ & Promega \\
\hline pRK2013 & Conjugation helper plasmid; $\mathrm{Kan}^{\mathrm{R}}$ & Figurski and Helinski (1979) \\
\hline pJQ200SK & sacB sacR Suc ${ }^{S}$; Gent ${ }^{R}$ & Quandt and Hynes (1993) \\
\hline рСРP30- & Broad host range; Tet ${ }^{R}$ & $\begin{array}{l}\text { Michael Kahn, Washington } \\
\text { State University }\end{array}$ \\
\hline pCPP30::aioX & $\begin{array}{l}\text { pCPP30 with } 1412 \text { bp fragment PCR cloned from strain } 5 \mathrm{~A} \text { containing } 304 \mathrm{bp} \\
\text { upstream sequence of aioX, whole aioX coding region and } 191 \mathrm{bp} \text { partial } \\
\text { aioS; Tet }{ }^{\mathrm{B}}\end{array}$ & This study \\
\hline pCPP30::Cys108Ser & $\begin{array}{l}\text { Site-directed mutagenesis of Cys-108 codon to Ser codon in aio } X \text { gene of } \\
\text { pCPP aioX; Tet }{ }^{\mathrm{P}}\end{array}$ & This study \\
\hline $\mathrm{pET}-52 \mathrm{~b}(+)$ & T7 RNA polymerase-based expression vector; $\mathrm{Amp}^{\mathrm{R}}$ & Novagen \\
\hline pETaioX3 & $\begin{array}{l}804 \mathrm{bp} \text { Ncol-Sacl fragment of aio } X \text { gene without signal sequence and stop } \\
\text { codon cloned into the multiple sites of } \mathrm{pET}-52 \mathrm{~b}(+) ; \mathrm{Amp}^{\mathrm{R}}\end{array}$ & This study \\
\hline pETaioX3C108A & $\begin{array}{l}\text { Site-directed mutagenesis of Cys-108 codon to Ala codon in aioX gene of } \\
\text { pET-52b(+); Amp }\end{array}$ & This study \\
\hline pETaioX3C108S & $\begin{array}{l}\text { Site-directed mutagenesis of Cys- } 108 \text { codon to Ser codon in aio } X \text { gene of } \\
\text { pET-52b(+); Amp }\end{array}$ & This study \\
\hline
\end{tabular}


total RNA, which was sevenfold that of Aslll-naïve cells (516 106 copies per nanogram of total RNA).

\section{Requirement of aioX for Aslll oxidation}

To investigate the role of aio $X$ in Aslll oxidation, a deletion mutation (nucleotides 12-861) was created in aioX (Fig. S3). Loss of AsIll oxidation in the $\triangle$ aio X mutant strain M53 was quantitatively demonstrated by HPLC-ICP-MS analysis of culture supernatants (Fig. 1). After $7 \mathrm{~h}$ growth in $100 \mu \mathrm{M}$ AsIll, growth profiles were similar for the different strains (Fig. 1A), although no Aslll oxidation was detected in mutant M53 or M53 carrying the control plasmid pCPP30 (Fig. 1B). In contrast, AsIll oxidation was observed with the wild-type strain and with M53 containing pCPP30::aioX (Fig. 1B).

\section{Association of AioX with the cytoplasmic membrane}

SignalP 3.0 software (http://www.cbs.dtu.dk/services/ SignalP) predicted the AioX $\mathrm{N}$-terminus contains a 39-amino-acid signal peptide with the twin-arginine translocation (TAT) motif SRRMAIG (Fig. S2). The signal peptide exhibits features consistent with it being an uncleaved signal anchor, with hidden Markov modelling predicting amino acids $1-20$ to be cytoplasmic (n-region),
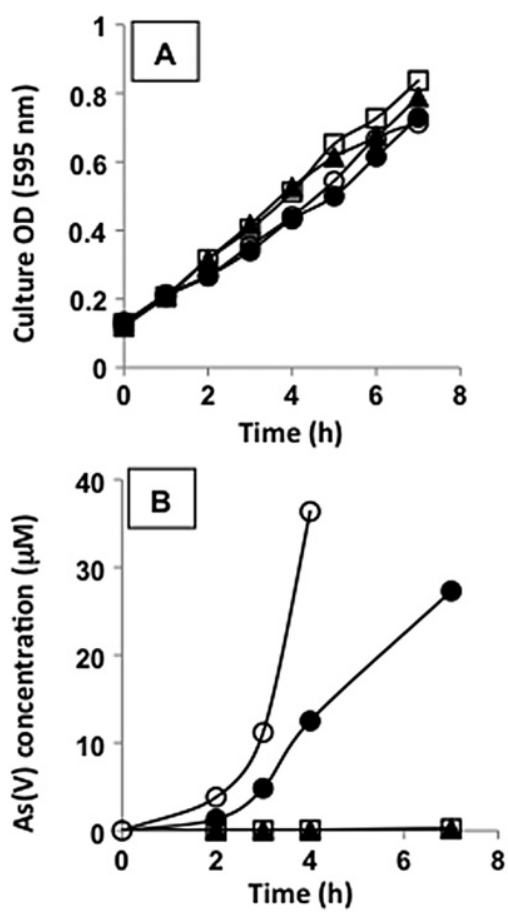

Fig. 1. Aslll oxidation properties of the wild-type strain $5 A$ and the $\Delta$ aioX mutant. Example of reproducible experiments illustrating: (A) growth profiles and (B) AsIll oxidation profiles in the presence of $100 \mu \mathrm{M}$ AsIll. ( $\bigcirc)$ wild-type strain 5A; ( $\square) \Delta$ aioX mutant M53; (O) M53 (pCPP30::aioX); and (А) M53 (pCPP30).

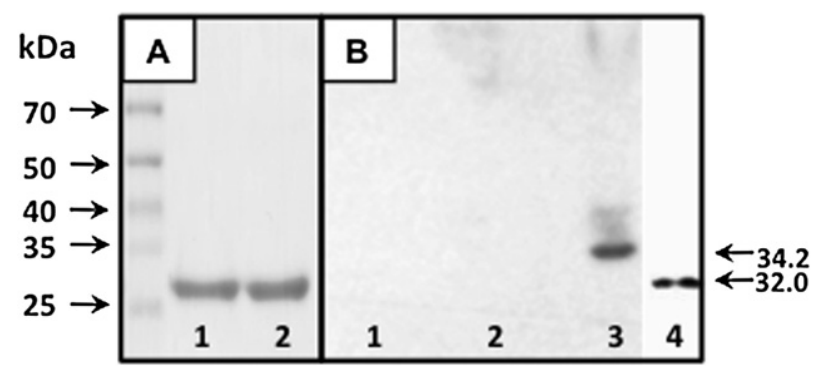

Fig. 2. Purification and Western blot analysis of AioX. A. Relative purity of the wild-type (lane 1) and Cys108Ser mutant (lane 2) versions of AioX purified without the signal peptide and with a His ${ }_{6}$ tag; $2.5 \mu \mathrm{g}$ of total protein was loaded in each well. B. Western blots of AsIII induced strain 5A total periplasm (lane 1), cytoplasm (lane 2) and cytoplasmic membrane (lane 3) extracts, and of purified AioX (lane 4, separate gel). Each cell extract fraction was loaded as $2.5 \mathrm{mg}$ total protein, whereas $0.25 \mu \mathrm{g}$ of purified AioX was used as positive control.

amino acids $21-43$ to be membrane spanning (h-region), and the balance of the protein (aa 44-306) to be periplasmic (c-region). Western blot analysis of cytoplasmic, periplasmic and cytoplasmic membrane-partitioned proteins with anti-AioX antibodies prepared against purified AioX (Fig. 2A) showed that AioX to be strictly associated with the cytoplasmic membrane (Fig. 2B). This is consistent with AioX being anchored to the cytoplasmic membrane in an $\mathrm{N}$-in/C-out orientation, i.e. a type II membrane protein (von Heijne, 1988). Note also that the apparent molecular weight of the monomer released from the solubilized membrane ( $\mathrm{MW}=34.2 \mathrm{kDa}$; Fig. $2 \mathrm{~B}$ ) is consistent with AioX retaining the signal peptide. To verify the cell fractionation procedure accurately reflects the targeted cell components, the above fractions were assayed for alkaline phosphatase (phosphate stressed cells), a known periplasmic marker protein expressed under phosphatestress conditions (Wanner, 1996). The periplasm fraction contained $77 \%$ of total alkaline phosphatase activity, illustrating that the periplasm fraction was enriched with periplasm proteins, although apparently lacking AioX.

\section{AioX is involved in regulation}

Since solute-binding proteins are often involved in regulation, the potential for AioX to be involved in Aslll-based regulation was then examined by quantitative RT-PCR analysis of expression of the Aslll oxidase structural genes aioBA (Fig. 3). In AsIll naïve cells, aioBA was not detected in either the wild-type or mutant strains (Fig. 3A), whereas in Aslll-treated cells the presence of aioBA mRNA was readily evident in the wild-type strain but absent in mutant M53 (Fig. 3A). The presence of pCPP30::aio $X$ converted the mutant back to wild type with respect to Aslll-based regulatory control of aioBA (Fig. 3B). 


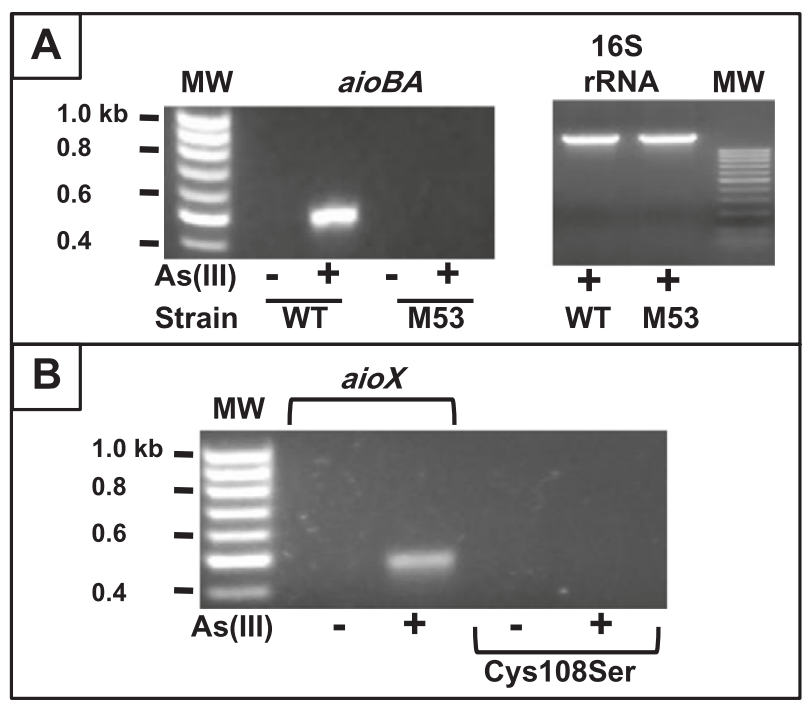

Fig. 3. Gene expression analysis of aioBA genes in wild-type strain $5 \mathrm{~A}$ and mutant M53.

A. RT-PCR analysis of aioBA expression in strains $5 \mathrm{~A}$ and M53 in cells exposed to $100 \mu \mathrm{M}$ AsIII (+) or Aslll-naïve (-). MW, molecular weight standards.

B. RT-PCR analysis of aioBA expression in mutant M53 complemented with wild-type aioX (pCPP30::aioX) or the Cys 108Ser mutated clone (pCPP30::Cys108Ser) in Aslll-treated (+) or untreated (-), early log-phase cultures. Equal volumes of all RT-PCR products were loaded in each lane.

The prediction of AioX being a solute-binding protein led us to compare AioX homologues to identify conserved cysteine residues because of their documented importance to AsIll interactions in Aslll-interactive proteins (Shi et al., 1994; Ruan et al., 2006; Lin et al., 2007). Of the four cysteines in AioX, only Cys 108 is completely conserved across all AioX-like proteins (Fig. S2), suggesting it is an essential feature. The Cys 108 was replaced with alanine or serine by site-directed mutagenesis and introduced to M53 via pCPP30. Neither mutant version rescued M53 with respect to appropriate regulation of aioBA (Fig. 3B) nor with respect to AsIll oxidation (Fig. S4), and was in contrast to the wild-type allele, showing that the conserved Cys108 in AioX is required for AsIll oxidation (Fig. S4).

\section{AioX binds AsIII}

Size exclusion chromatography determined the $\mathrm{His}_{6}{ }^{-}$ tagged AioX native protein molecular weight to be $32 \mathrm{kDa}$ (Fig. 4) (30 kDa without the $\mathrm{His}_{6}$-tag), suggesting AioX in these preparations was a monomer. AioX samples incubated with AsIII prior to gel filtration behaved differently (Fig. 4), exhibiting slightly earlier elution and a reproducible minor shoulder. This suggested that AioX interacts with AsIll, resulting in a conformational change and causing AioX to behave as if it had increased in size to $\sim 33.7 \mathrm{kDa}$ (Fig. 4).
ICP-MS analysis was then used to directly demonstrate AsIll binding to AioX. AioX was incubated with excess Aslll for $1 \mathrm{~h}$ and then passed through a desalting column to separate unbound AsIII from the AioX-AsllI complex. As expected, the protein eluted first, primarily in fractions $1-3$, with the Aslll breakthrough observed in fractions 5 or 6. Minimal background AsIII in the reagents provided a weak ICP-MS signal with AioX pre-incubated in buffer only, whereas fractions containing AioX pre-incubated with AsIll contained $0.327 \pm 0.029 \mu \mathrm{mol}$ AsIll per $\mu \mathrm{mol}$ AioX (Fig. 5A). With the Cys108Ser mutant, AsIll binding was significantly lower (approximately sixfold reduction; $0.055 \pm 0.008 \mu \mathrm{mol}$ AsIll per $\mu \mathrm{mol}$ AioX) (Fig. 5A).

Fluorescence quenching titration was performed to determine the stoichiometry and dissociation constant of the AsIII binding. The tryptophan-based intrinsic fluorescence of AioX was quenched by AsIII in a dose-dependent manner, with quenching nearly saturated at $20 \mu \mathrm{M}$ AsIII (Fig. 5B). The titration data of the fluorescence quenching fits the model of one specific binding site (Fig. 5B insert), yielding a $K_{\mathrm{D}}$ value of $2.4 \pm 0.2 \mu \mathrm{M}$ for the AioX-AsIII complex. In contrast, $20 \mu \mathrm{M}$ AsIll failed to quench the intrinsic fluorescence of AioX ${ }^{\text {C108S }}$ (Fig. 5B). The significantly reduced tryptophan spectra suggested the Cys108Ser amino acid change resulted in a conformational change that significantly altered the microenvironment of the nearby tryptophan (W106), which is also highly conserved (Fig. S2).

The relatively low stoichiometry of 0.33 mole AsIII per mole AioX (Fig. 5A) prompted additional experiments aimed at better understanding what appears at this stage

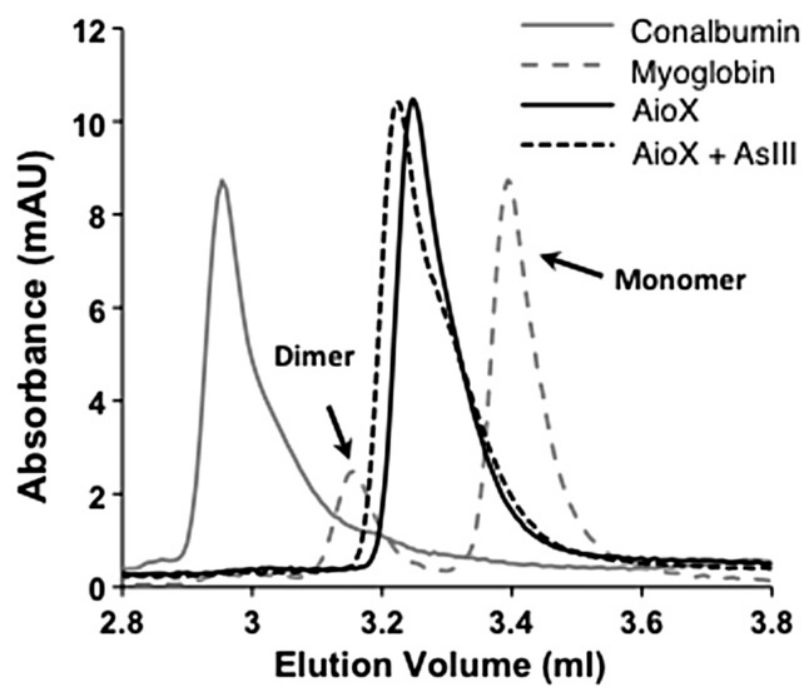

Fig. 4. Gel filtration chromatographic elution profile of AioX $(1.0 \mu \mathrm{M})$ with or without pre-incubation with $20 \mu \mathrm{M}$ AsIll. Protein standards used to differentiate between monomer versus dimer forms of AioX were conalbumin ( $\mathrm{MW}=75 \mathrm{kDa}$ ) and myoglobin $($ monomer $=16.7 \mathrm{kDa}$, dimer $=33.4 \mathrm{kDa})$. 

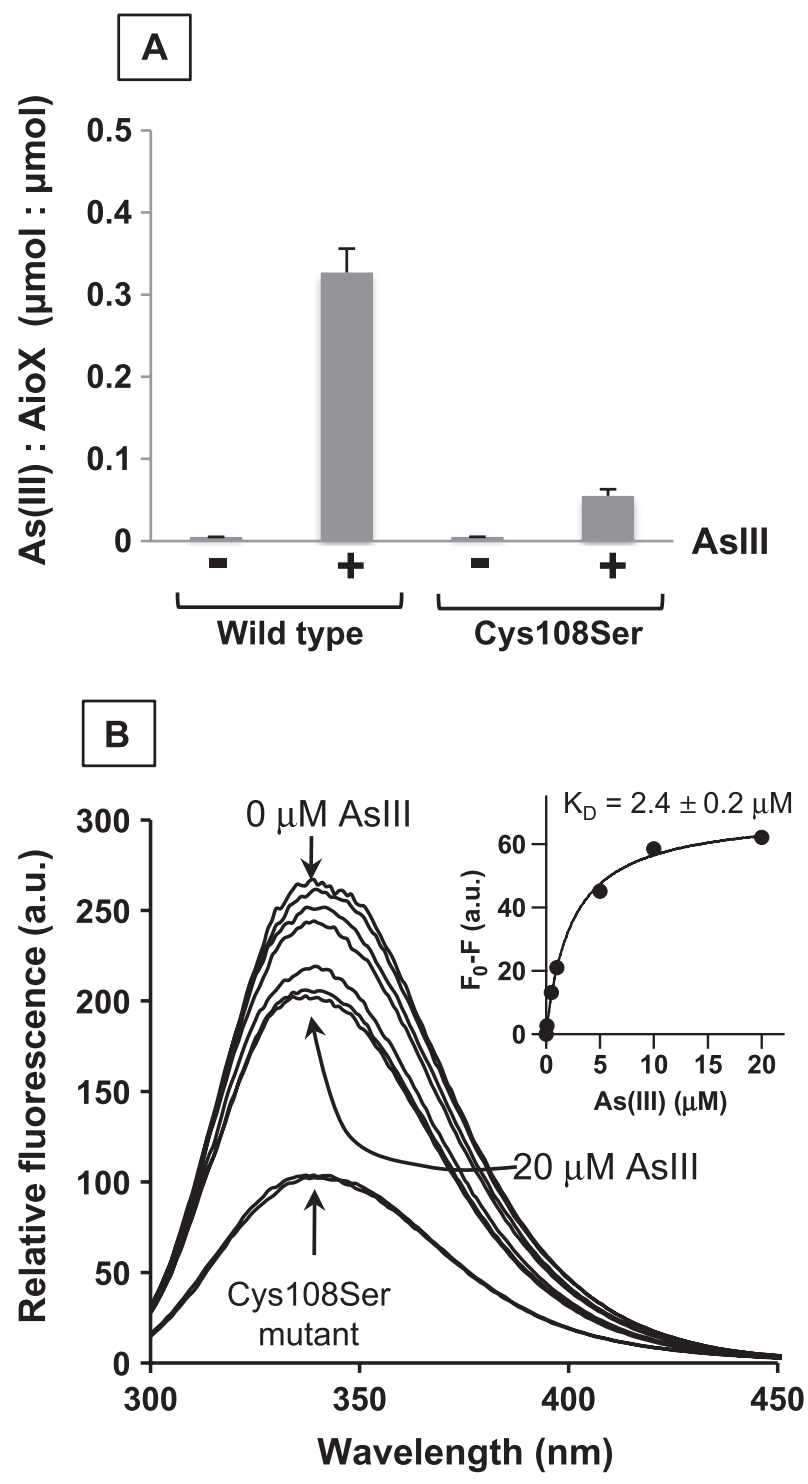

Fig. 5. Evidence of AioX binding AsIll.

A. Wild-type and the Cys108Ser mutant protein samples were pre-incubated in Tris buffer, $\mathrm{pH} 7.5$ with $20 \mu \mathrm{M}$ AsllI (+) or in Tris buffer alone as a control (-). Data are representative and are the average \pm standard error (error bars) of the first two fractions eluting from the desalting column. Units: $\mu \mathrm{mol}$ AsIll per $\mu \mathrm{mol}$ protein.

B. Tryptophan-derived intrinsic fluorescence spectra of the wild-type and Cys108Ser mutant proteins. Relative fluorescence of wild-type AioX samples $(1.0 \mu \mathrm{M})$ was measured as a function of incremental increases of AsllI (0, 0.1, 0.5, 1.0, 5.0, 10 and $20 \mu \mathrm{M})$.

Fluorescence of the Cys108Ser mutant version was significantly muted and did not change when AsIll concentration was increased from 0 to $20 \mu \mathrm{M}$. Fluorescence change at $340 \mathrm{~nm}$ was analysed by GraphPad Prism 5, and $K_{\mathrm{D}}$ value was calculated to be $2.4 \pm 0.2 \mu \mathrm{M}$.

to be a novel AsIII binding mechanism. The relatively low stoichiometry could result from the AsIll binding site being partially occluded or from disassociation of Aslll during transit of the protein through the desalting column, imply- ing AsIII binding is readily reversible. The latter was examined in competitive binding experiments between AsIII and the dye BODIPY® 577-618 maleimide, which forms sulfhydryl-specific covalent bonds. With $1.0 \mu \mathrm{M}$ AioX preincubated with $2 \mu \mathrm{M}$ AsIII for $1 \mathrm{~h}$, introduction of equimolar concentrations of dye resulted in increasing levels of dye bonded to AioX over time (Fig. 6). In the absence of a competing ligand, dye binding to AioX proceeded more rapidly and to a greater extent (Fig. 6).
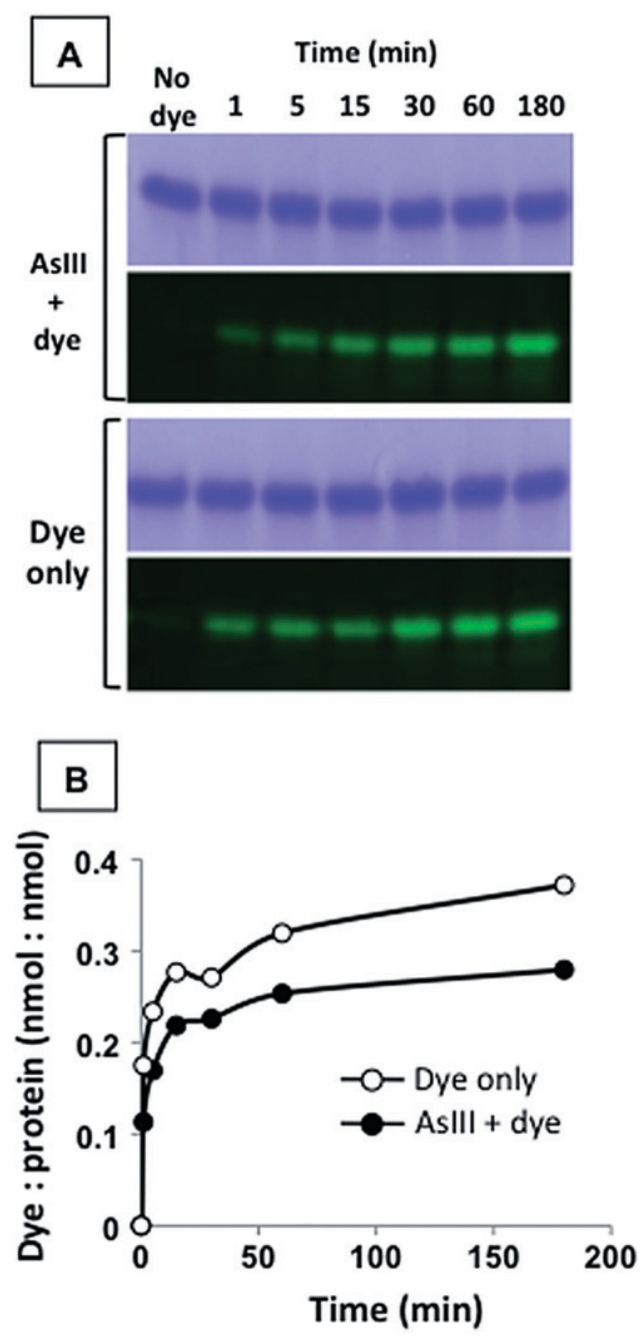

Fig. 6. Competitive ligand binding with AioX.

A. Coomassie blue protein staining (blue bands) illustrates uniform protein loading and fluorescence scans (false green colouring) of the same AioX protein bands exhibiting different degrees of dye binding as a function of pre-incubation with AsIll. Purified AioX was pre-incubated with $2 \mu \mathrm{M}$ AsIll for $1 \mathrm{~h}$ followed by $2 \mu \mathrm{M}$ dye

(AsIII + dye), or only with $2 \mu \mathrm{M}$ dye without AsIll pre-incubation (Dye only). At specified times, $10 \mu \mathrm{l}$ aliquots were removed and $200 \mathrm{mM}$ DTT added to stop the reaction.

B. Protein content and dye binding in SDS-PAGE gels was quantified by TotalLab Quant image analysis software. 


\section{Discussion}

This study describes a significantly novel development in our understanding of how microbe-arsenic interactions are regulated. Heretofore, only the repressor ArsR and the AsIll metallochaperone ArsD proteins involved in regulating arsenic detoxification have been shown to specifically interact with AsIll via distinct cysteine residues (Bhattacharjee and Rosen, 2007). The frequent occurrence of aioX in known Aslll-oxidizing microbes (Fig. S1) and its close physical proximity to other genes known to be essential to Aslll oxidation (Fig. S1) suggested aioX encodes a protein important for AsIll oxidation. The present study confirmed the essential nature of this gene (Fig. 1B, Fig. S4), and that it encodes a protein that is critical for normal regulatory control of genes also known to be essential to Aslll oxidation (Figs 1 and 3).

Annotation, protein modelling exercises and amino acid alignments of AioX with its homologues suggested this protein is located to the periplasm, being translocated via a TAT signal peptide (Fig. S2). However, Western blot evidence showed that AioX was exclusively associated with the cytoplasmic membrane and electrophoresed as a $34.1 \mathrm{kDa}$ peptide (Fig. 2), arguing that the signal peptide remains uncleaved. As such, this provides experimental support to suggest that AioX is a Gramnegative signal anchor protein, for which there are very few examples (Nielsen and Krogh, 1998; Brunak et al., 2007) and thus another novel observation deriving from this investigation.

When expressed and characterized without the signal peptide (to afford purification), AioX was found to behave as a monomer (Fig. 4) and thus is consistent with descriptions of other characterized periplasmic solute-binding proteins (Lever, 1972; De Pina et al., 1995; He et al., 2009). Evidence of Aslll binding is threefold: (i) in size exclusion chromatography experiments, AioX eluted as a monomer, although pre-incubation with AsIll altered AioX behaviour in the gel matrix, indicating that Aslll binding effects a conformational change in AioX (Fig. 4), (ii) this conformation change is consistent with reproducible shifts in the intrinsic fluorescence profile with incremental increases in added AsIII, yielding a $K_{\mathrm{D}}$ estimate of $2.4 \mu \mathrm{M}$ AsIII (Fig. 5B), and (iii) direct binding assays illustrated AsIII co-eluting with AioX and separate from the AsIII breakthrough (Fig. 5A).

Substitution of the highly conserved Cys 108 resulted in complete loss of Aslll oxidation (Fig. S4), lack of aioBA induction (Fig. 3B), and collapse of Aslll binding (Fig. 5A and B). An AsIll : AioX stoichiometry less than unity is perhaps explained by AsIll disassociating from AioX during transit in the column, although we draw attention to the similar stoichiometry for the sulfhydryl-specific dye used in the ligand binding competition experiments that demonstrated the potential for AsIll to disassociate from AioX (Fig. 6). The exact mechanism of AsIll binding presumably involves Cys108, although we note this substantially deviates from the current AsIII-binding paradigm that always involve proteins (ArsR and ArsD) employing multiple cysteines and typically as homodimers (Bhattacharjee and Rosen, 2007). Potentially, AioX may actually function as a multimer while anchored to the membrane. Current efforts are underway to further elucidate AioX form and function of Aslll binding.

Our previous efforts yielded a fairly simple model for explaining Aslll oxidation regulation (Kashyap et al., 2006a). AsIll was viewed to be detected by the sensor kinase AioS, which then phosphorylates its cognate response regulator AioR that then actuates the upregulation of essential genes such as aioBA (Kashyap et al., 2006a). A recent study by Sardiwal and colleagues (2010) demonstrated AioR being phosphorylated by a component of Aios and thus supports this model. However, the experiments reported herein illustrate that this above model is too simple. The $\Delta$ aio $X$ mutant was totally devoid of aioBA expression (Fig. 3), implying AioX plays an essential role, likely either in sensing or in transducing the AsIll signal. Given the combined evidence showing AioX to be a membrane bound exported protein (Fig. S2, Fig. 2) capable of binding AsIII (Figs 4-6), it is reasonable at this juncture to conclude that AioX is a periplasm located Aslll-binding protein.

The role of periplasmic solute-binding proteins in regulating gene expression via two-component systems is well supported by the literature (reviewed by Tam and Saier, 1993; Mascher et al., 2006). This class of proteins is sometimes involved in initializing solute transport, such as PstS in high-affinity phosphate transport (Luecke and Quiocho, 1990). In preliminary experiments, we as yet have been unable to show that AioX is required for Aslll uptake. Given the regulatory phenotype of the $\Delta$ aioX mutant (Fig. 3) and the properties of AioX (Figs 2-5 and Fig. S2), our current working model is that AioX actually functions as the Aslll sensor, with AsIll binding resulting in a conformational change that then facilitates intramolecular communication with the sensor kinase AioS. This would be similar to the A.tumefaciens glucose-binding protein, ChvE, which mediates the sugar-induced virulence response governed by the VirA/ VirG two-component signal transduction system (He et al., 2009).

In summary, the combination of experiments summarized herein support an updated model that now includes AioX as part of an apparent three component system that involves AioX binding AsIII, and that then initiates signal transduction by either AsIll being transferred to AioS or an AioX conformational change causing AioX to then specifi- 
cally interact with Aios to facilitate the same signalling pathway. Accordingly, molecular and genetic regulatory models for microbial AsIll oxidation should now reflect a more sophisticated system.

\section{Experimental procedures}

\section{Bacterial strains and growth conditions}

Bacterial strains and plasmids used in this study are listed in Table 1. The A. tumefaciens strains were grown at $30^{\circ} \mathrm{C}$ in a defined minimal mannitol ammonium (MMN) medium as described previously (Somerville and Kahn, 1983), but modified to contain $50 \mu \mathrm{M}$ phosphorus. Escherichia coli strains were cultured at $37^{\circ} \mathrm{C}$ in Luria-Bertani medium (Sambrook et al., 1989). Kanamycin $\left(25 \mu_{\mathrm{g} \mathrm{ml}}{ }^{-1}\right)$, gentamicin $\left(25 \mu \mathrm{g} \mathrm{ml}^{-1}\right)$, tetracycline $\left(10 \mu \mathrm{g} \mathrm{ml}^{-1}\right)$, chloramphenicol $\left(34 \mu \mathrm{g} \mathrm{m}^{-1}\right)$ or ampicillin $\left(100 \mu \mathrm{g} \mathrm{ml}^{-1}\right)$ were added as needed. Isopropyl $\beta$-D-1-thiogalactopyranoside (IPTG, $0.4 \mathrm{mM}$ ) and 4-chloro-3-indoyl- $\beta$-D-galactoside (X-Gal, $40 \mathrm{mg}$ per litre of agar medium) were added as required for detecting PCR amplicons cloned into the pGEM-T vector.

\section{Identification of the aioX gene}

Thermal asymmetric interlaced polymerase chain reaction (TAIL-PCR) was performed to identify the complete aioX gene in strain $5 \mathrm{~A}$ as described previously by Liu and Whittier (1995). Three specific primers (5ALB1, 5'-CTTTCC CGCTGTCGTG-3', 5ALB2, 5'-GAGAGCAGTTCCGGTTC TG-3' and 5ALB3, 5'-GGCCAGATAGGTCTTCGTGAC-3') complementary to the sequences flanking the aioSRBA locus (GenBank Accession No. DQ151549) were used in combination with short arbitrary, degenerate primers (Liu and Whittier, 1995). After TAIL-PCR, all products were gel-purified, subcloned into pGEM-T and sequenced. Multiple inferred amino acid sequence alignments of putative AioX homologues obtained from NCBI database were performed with CLUSTALX 2.0 (Thompson et al., 1997).

\section{Creation of aioX mutants and complementation}

A deletion mutation was introduced into the aio $X$ coding region using cross-over PCR. Two separate PCRs were performed with primer pairs: $\triangle$ aioX1 [GGATCCGCGACAGGT GCGGATGA] with $\triangle$ aioX2 [CCCATCGATTAAACTTAAACA CGATTTGACACCGACGACCTCCCTC] and $\triangle$ aioX3 [TGT TTAAGTTTAATCGATGGGCCACCCGAAAGCTACGA] with $\triangle$ aioX4 [GGATCC CGAAAGAGGCGTGTATGGTCCCGAT] (BamHI restriction sites in bold) to generate fragments upstream and downstream of the DNA targeted for deletion. The resulting products were mixed and used as template for cross-over PCR with primers $\Delta a i o \mathrm{X} 1$ and $\Delta a i o \mathrm{X} 4$. The two fragments were capable of annealing to each other by the $21 \mathrm{bp}$ complementary tag sequence (underlined in the primers $\triangle a i o X 2$ and $\Delta a i o X 3)$. The generated fusion fragment of $\sim 1400 \mathrm{bp}$ was subcloned into BamHI-digested pJQ200SK, resulting in pJQ200SK $\Delta$ aioX, which was then mobilized into strain $5 \mathrm{~A}$ by the conjugative E. coli HB101
(pRK2013). The wild-type aioX allele was then replaced by the $\Delta$ aio $X$ gene using levansucrase selection as we previously described (McDermott and Kahn, 1992). Gm merodiploid transconjugants were selected on minimal mannitol agar and then transconjugants were subcultured onto minimal mannitol-15\% sucrose agar. Sucrose ${ }^{R} \mathrm{Gm}^{\text {Sen }}$ transconjugants were then screened using diagnostic PCR to identify a double recombinant, followed by sequencing of the amplicons to verify that the correct mutation had been introduced. The resulting $\triangle$ aio $X$ mutant is referred to as strain M53.

A Cys108Ala mutation and a Cys108Ser mutation were introduced using a QuikChange II XL Site-Directed Mutagenesis Kit (Stratagene). The Cys108 residue was changed to Ala (C108A) using the primers (EHKC108AF, GGCCGCCT GGATTGCGGGCTACCCGTTCATG and EHKC108AR, CA TGAACGGGTAGCCCGCAATCCAGGCGGCC). The change to serine used primers EHKC108SF (5'-GGCCGCCTGGAT TTCTGGCTACCCGTTCATG-3') and EHKC108SR (5"-CA TGAACGGGTAGCCAGAAATCCAGGCGGCC-3'). Plasmid DNA was isolated with the QIAprep $®$ Spin Miniprep Kit (Qiagen). Restriction endonuclease digestion, DNA purification, ligation and transformation were performed with the manufacturer's standard protocols. All putative mutations were confirmed by DNA sequencing.

\section{PCR and DNA sequencing}

For mutant complementation, a 1412 bp fragment containing the complete aioX coding region along with 304 bp upstream sequence and $191 \mathrm{bp}$ downstream DNA (including part of downstream adjacent aioS) was PCR-amplified using primers [CGGCCGGGTGGTAGCGAGCGAAAT, Pstl site in bold and GAATTCGGGATAAGAGCGGTAGACAA, EcoRI in bold], and subcloned into Pstl+EcoRI-digested pCPP30. The resulting plasmid was transferred into the mutant M53 by conjugation (as above).

\section{Gene expression analyses}

Reverse transcriptase PCR (RT-PCR) and quantitative (q)RT-PCR were applied to assess transcription of the genes described in this study. Total RNA was extracted from strains $5 \mathrm{~A}$ or M53 grown with and without $100 \mu \mathrm{M}$ AsIll using RNeasy ${ }^{\circledR}$ Mini Kit (Qiagen), then treated with DNase using Turbo DNA-free (Ambion, Austin, TX) and purified using the Ambion MEGAclear kit (Ambion, Austin, TX) following the manufacturer's instructions. RNA preparations were verified to be free of DNA by PCR. Primers aioXRTF $\left(5^{\prime}\right.$-TCATA CCTCATCGTCGGTCA-3') and aioXRTR (5'-GAGCGCGT TTCTTATTCTGG-3') were designed for routine RT-PCR monitoring of aioX. Primers P4 and P5 (described in Kashyap et al., 2006a) were used for detecting expression of aioBA. The RT-PCRs were performed using the Access Quick RT-PCR system (Promega) following the manufacturer's recommended protocol. The annealing temperature for the aioX RT-PCRs was $53^{\circ} \mathrm{C}$. For qRT-PCR, primer XrealF (5'-TG GATACGTCTGGGAAGTCATG-3') paired with XrealR (5'GCGTTTCTTATTCTGGCAACC-3') were used. For RTPCRs involving the 16S rRNA, primers $8 \mathrm{~F}$ and $1392 \mathrm{R}$ were used as we have previously described (Kashyap et al., 
2006a). For qRT-PCR, $10 \mathrm{ng}$ of total RNA was first reverse transcribed by M-MLV Reverse Transcriptase (Ambion, Austin, TX). The resulting cDNA was then used as template for qPCR with the GoTaq ${ }^{\circledR}$ qPCR Master Mix (Promega). The standard curves in quantitative RT-PCR were generated using plasmid pGEM-T containing cloned target sequences.

\section{Plasmid construction, expression and purification of AioX proteins}

The AioX wild-type and mutant proteins were expressed in E. coli BL21 Star ${ }^{\mathrm{TM}}(\mathrm{DE} 3)$ pLysS with aioX genes on vector $\mathrm{pET}-52 \mathrm{~b}(+)$. The aio $X$ coding region without the $117 \mathrm{bp}$ signal peptide sequence was PCR cloned by Pfx50 ${ }^{\mathrm{TM}}$ DNA Polymerase (Invitrogen) with primers 5'-CCATGGGCGA GTTGCTGTCCGTG-3' (Ncol site in bold) and 5'-GAGCTC CCCTAGCCTCCGAACAC-3' (Sacl site in bold), and subcloned into $\mathrm{Ncol}+$ Sacl-digested sites of $\mathrm{pET}-52 \mathrm{~b}(+)$, resulting in pETaioX3 or pETaioX3Cys108Ser, each with a C-terminal his tag. Cells were grown at $37^{\circ} \mathrm{C}$ overnight in $50 \mathrm{ml}$ of M9ZB medium (Studier, 1991) containing the required antibiotics and transferred into $3 \mathrm{I}$ of fresh media with the same antibiotics. Cells were induced at an $\mathrm{OD}_{600}$ of $\sim 0.5$ by adding IPTG, harvested by centrifugation (7000 $\mathrm{g}$ for $10 \mathrm{~min}$ at $4^{\circ} \mathrm{C}$ ) after induction (overnight for the wild-type protein and $6 \mathrm{~h}$ induction for mutant protein), and suspended in $20 \mathrm{mM}$ Tris- $\mathrm{HCl}$, $\mathrm{pH}$ 8.0. After washing with $\mathrm{Tris}-\mathrm{HCl}$, the pellets were lysed via sonication on ice for $10 \mathrm{~min}$. Unbroken cells were removed by centrifugation at $12000 \mathrm{~g}$ for $20 \mathrm{~min}$. The soluble supernatant was mixed with I $\mathrm{ml}$ of pre-equilibrated TALON® Metal Affinity Resins (Clontech) and gently agitated at $4^{\circ} \mathrm{C}$ for $30 \mathrm{~min}$ on a platform shaker to allow the polyhistidine-tagged protein to bind the resin. The resin was transferred to a $2 \mathrm{ml}$ gravity-flow column and washed by $10 \mathrm{ml}$ of Tris- $\mathrm{HCl}$, then eluted with a linear gradient of $0.005-0.5 \mathrm{M}$ imidazole in Tris- $\mathrm{HCl}, \mathrm{pH}$ 8.0. Fractions were collected, analysed by SDS-PAGE and verified to be AioX by Matrix Assisted Laser Desorption/lonization Time-of-Flight (MALDI-TOF) Mass Spectrometry. Fractions containing a single band corresponding to the $32 \mathrm{kDa}$ polyhistidine-tagged protein was observed with $>95 \%$ purity and dialysed against 3 I of $20 \mathrm{mM}$ Tris- $\mathrm{HCl}(\mathrm{pH} 8.0)$ overnight at $4^{\circ} \mathrm{C}$. An additional clean-up step was included to remove some non-target proteins that bound to the resin. Proteins were loaded into DEAE Sepharose Fast Flow Column (GE Healthcare), which was pre-equilibrated with Tris- $\mathrm{HCl}, \mathrm{pH} 8.0$ and eluted with a linear gradient of $0-0.5 \mathrm{M} \mathrm{NaCl}$. The AioX was pooled and stored at $-80^{\circ} \mathrm{C}$ until use. Protein concentration was determined using Pierce ${ }^{\circ}$ BCA Protein Assay Kit (Thermo Scientific).

\section{Measurement of arsenite binding: (i) Aslll-protein association}

Purified wild type and the Cys108Ser mutant AioX were incubated with AsIll (AsIll : AioX molar ratio of 2:1) at room temperature for $1 \mathrm{~h}$. The protein-AsIll mixture was passed through a Sephadex® G-25 (fine) desalting column (GE Healthcare) that had been pre-equilibrated with $20 \mathrm{mM}$ Tris$\mathrm{HCl}, \mathrm{pH}$ 8.0. Eluted fractions were analysed for protein via the Bradford assay and for AsIll by inductively coupled mass spectrometry (ICP-MS) (Agilent 7500ce).

\section{Measurement of arsenite binding: (ii) fluorescence spectroscopy}

Fluorescence measurements were performed with a Cary Eclipse Fluorescence Spectrophotometer (Varian Scientific Instruments, Mulgrave, Australia) at room temperature. Tryptophan fluorescence was monitored with an excitation wavelength of $280 \mathrm{~nm}$ and the emission scans were conducted between 300 and $500 \mathrm{~nm}$. The wild-type and mutant proteins were $1.0 \mu \mathrm{M}$ in $20 \mathrm{mM}$ Tris- $\mathrm{HCl}(\mathrm{pH} 8.0)$, unless otherwise noted. The fluorescence of Tris-HCl buffer alone or with AsIII was performed as blank control between scanning of duplicate samples for each Aslll concentration used.

\section{Size exclusion chromatography}

Size exclusion chromatography was performed on an ÄKTA FPLC system (Amersham Pharmacia Biotech) using a Agilent Bio SEC-3 Column ( $3 \mu \mathrm{m}, 300 \AA$, $4.6 \mathrm{~mm} \times 300 \mathrm{~mm}$ ). The mobile phase contained $20 \mathrm{mM}$ Tris- $\mathrm{HCl}(\mathrm{pH} 7.5)$ with $150 \mathrm{mM} \mathrm{NaCl}$ and was run at a flow rate of $0.2 \mathrm{ml} \mathrm{min}^{-1}$. The protein concentration was measured using UV absorbance at $280 \mathrm{~nm}$. Two standard proteins (conalbumin, $75 \mathrm{kDa}$ and myoglobin, $16.7 \mathrm{kDa}$ ) were used to estimate molecular mass based on elution volume, primarily to distinguish between the monomer and dimmer forms of AioX.

\section{Cell extract preparation}

The periplasmic, cytoplasmic and membrane proteins were fractionated from AsIll-exposed cells based on the procedure of De Maagd and Lugtenberg (1986) with modification. In brief, in addition to adding $100 \mu \mathrm{M}$ AsIll, the cells were starved for phosphate in order to induce alkaline phosphatase, which was the periplasmic marker enzyme. The cells were pelleted by centrifugation, resuspended in $5 \mathrm{ml}$ of suspension buffer (200 mM Tris- $\mathrm{HCl}, \mathrm{pH}$ 8.0, $0.5 \mathrm{M}$ sucrose, $1 \mathrm{mM}$ EDTA, $1 \mathrm{mM}$ phenylmethylsulfonyl fluoride) supplemented with $0.2 \mathrm{mg}$ of lysozyme per $\mathrm{ml}$, and incubated at room temperature for $30 \mathrm{~min}$. The cells were pelleted by centrifugation at $4^{\circ} \mathrm{C}$ and the supernatant was saved as the periplasmic fraction. The resulting pellet contained spheroplasts. To isolate the cytoplasmic proteins, the spheroplasts were lysed by suspension in $5 \mathrm{ml}$ of chilled double-distilled water, followed by brief sonication. Unspheroplasted cells were removed by low-speed centrifugation. The supernatant was reserved as the cytoplasmic + cytoplasmic membrane fractions which were further fractionated by ultracentrifugation (262 $000 \mathrm{~g}$ for $1 \mathrm{~h}$ at $4^{\circ} \mathrm{C}$ ) to pellet the membranes. The supernatant was reserved as the cytoplasmic fraction. The membrane pellet was then dissolved in SDS sample buffer and kept as the membrane fraction. Proteins of the supernatant (periplasmic and cytoplasmic fractions) were concentrated by acetone precipitation. Alkaline phosphatase (AP) activity was measured by recording the hydrolysis of p-nitrophenyl phosphate (Bessey et al., 1946). Briefly, $10 \mu \mathrm{l}$ 
of the different fractions were mixed well with $280 \mu \mathrm{l}$ of buffer A (300 mM 2-amino-2-methyl-1, 3-propanediol, $2 \mathrm{mM}$ $\left.\mathrm{MgCl}_{2}, \mathrm{pH} 10.25\right)$ and $10 \mu \mathrm{l}$ of substrate buffer $(400 \mathrm{mM}$ 4-nitrophenyl phosphate). The hydrolysis of p-nitrophenyl phosphate was measured spectrophotometrically at $405 \mathrm{~nm}$ and $37^{\circ} \mathrm{C}$ with a SpectraMax Plus ${ }^{384}$ UV/Vis spectrophotometer (Molecular Devices, Sunnyvale, CA). One unit of the AP activity was defined as catalysing 1 micromole of p-nitrophenyl phosphate per minute per milligram of protein at $37^{\circ} \mathrm{C}$.

\section{Mouse immunization and Western immunoblot analysis}

Two female BALB/c mice (4 weeks old; from National Cancer Institute, Frederick Animal Production Area, Frederick, MD) were immunized subcutaneously and then boosted with $30 \mu \mathrm{g}$ of purified recombinant AioX suspended in $160 \mu \mathrm{l}$ of aluminium hydroxide gel (Sigma) on days 1 and 14 . Immune antiserum was collected 2 weeks after the second boost.

Western immunoblot analysis was performed to detect the location of the AioX as described (Lei et al., 2004). Equal amounts of fractions and purified recombinant proteins were resolved by SDS-PAGE and transferred to nitrocellulose membranes (BioTrace ${ }^{T M}$ NT, Pall Corporation) with Towbin transfer buffer using a Trans-Blot SD semidry transfer cell (Bio-Rad) at $15 \mathrm{~V}$ for $45 \mathrm{~min}$. The membrane was blocked with 1:20 Amersham Liquid Block in Tween buffer $(0.1 \%$ Tween 20 in PBS) for $1 \mathrm{~h}$ and subsequently incubated for $1 \mathrm{~h}$ with anti-AioX mouse antiserum added to the block solution (1:500 dilution). The membrane was then rinsed twice and washed three times for 15 min each with $0.1 \%$ Tween 20 in PBS. The membrane was incubated for $1 \mathrm{~h}$ with goat antimouse IgG-HRP (1:2000 dilution, Santa Cruz Biotechnology) in the block solution and rinsed and washed as described above. Antibody-antigen interaction was visualized by enhanced chemiluminescence.

\section{Binding-competition assays}

The thiol-reactive dye BODIPY® 577/618 maleimide (Invitrogen) was used to demonstrate the reversible binding between AioX and AsIII. AioX and AsIII (2:1 molar ratio) were incubated for $1 \mathrm{~h}$ incubation at room temperature, followed by dye addition (1:1, AsIll : dye ratio). The binding was stopped by adding $200 \mathrm{mM}$ DTT immediately. Subsequently, the proteins were analysed by SDS-PAGE and gels were scanned at excitation/emission maxima of $\sim 577 / 618 \mathrm{~nm}$ using the Typhoon Trio Variable Mode Imager (GE Healthcare). All gels were quantified using TotalLab Quant image analysis software (http://www.totallab.com/products/totallabquant).

\section{Acknowledgements}

This research was supported by a Major International Joint Research Project of Chinese National Natural Science Foundation (31010103903) to G.W. (Co-PIs, T.R.M. and C.R.) and by the US National Science Foundation to T.R.M. and B.B. (MCB 0817170). G.L. was supported by the PhD student exchange scholarship of the Ministry of Education, China. The authors thank B.P. Rosen for stimulating discussion.

\section{References}

Afkar, E., Lisak, J., Saltikov, C., Basu, P., Oremland, R.S., and Stolz, J.F. (2003) The respiratory arsenate reductase from Bacillus selenitireducens strain MLS10. FEMS Microbiol Lett 226: 107-112.

Anderson, C.L., Williams, J., and Hille, R. (1992) The purification and characterization of arsenite oxidase from Alcaligenes faecalis, a molybdenum-containing hydroxylase. J Biol Chem 267: 23674-23682.

Bessey, O.A., Lowry, O.H., and Brock, M.J. (1946) A method for the rapid determination of alkaline phosphatase with five cubic millimeters of serum. J Biol Chem 164: 321-329.

Bhattacharjee, H., and Rosen, B.P. (2007) Arsenic metabolism in prokaryotic and eukaryotic microbes. In Molecular Microbiology of Heavy Metals. Nies, D.H., and Silver, S. (eds). Heidelberg, Germany: Springer, pp. 371-406.

Boyer, H.W., and Roulland-Dussoix, D. (1969) A complementation analysis of the restriction and modification of DNA in Escherichia coli. Mol Biol 41: 459-472.

Brunak, S., Emanuelsson, O., Nielsen, H., and von Heijne, G. (2007) Locating proteins in the cell using TargetP, SignalP and related tools. Nat Protoc 2: 953-971.

Cai, L., Rensing, C., Li, X., and Wang, G. (2009) Novel gene clusters involved in arsenite oxidation and resistance in two arsenite oxidizers: Achromobacter sp. SY8 and Pseudomonas sp. TS44. Appl Microbiol Biotechnol 83: 715-725.

Cullen, W.R., and Reimer, K.J. (1989) Arsenic speciation in the environment. Chem Rev 89: 713-764.

De Maagd, R.A., and Lugtenberg, B. (1986) Fractionation of Rhizobium leguminosarum cells into outer membrane, cytoplasmic membrane, periplasmic, and cytoplasmic components. J Bacteriol 167: 1083-1085.

De Pina, K., Navarro, C., McWalter, L., Boxer, D.H., Price, N.C., Kelly, S.M., et al. (1995) Purification and characterization of the periplasmic nickel-binding protein NikA of Escherichia coli K12. Eur J Biochem 227: 857-865.

Ellis, P.J., Conrads, T., Hille, R., and Kuhn, P. (2001) Crystal structure of the $100 \mathrm{kDa}$ arsenite oxidase from Alcalingenes faecalis in two crystal forms at $1.64 \AA$ and $2.03 \AA$. Structure 9: 125-132.

Figurski, D.H., and Helinski, D.R. (1979) Replication of an origin-containing derivative of plasmid RK2 dependent on a plasmid function provided in trans. Proc Natl Acad Sci USA 76: 1648-1652.

Hanahan, D. (1983) Studies on transformation of Escherichia coli with plasmids. J Mol Biol 166: 557-580.

He, F., Nair, G.N., Soto, S.C., Chang, Y., Hsu, L., DeGrado, W.F., and Binns, A.N. (2009) Molecular basis of ChvE function in sugar binding, sugar utilization, and virulence in Agrobacterium tumefaciens. J Bacteriol 191: 58025813.

von Heijne, G. (1988) Transcending the impenetrable: how proteins come to terms with membranes. Biochim Biophys Acta 947: 307-333.

Inskeep, W.P., McDermott, T.R., and Fendorf, S.E. (2001) Arsenic (V)/(III) cycling in soils and natural waters: chemical and microbiological processes. In Environmental Chemistry of Arsenic. Frankenberger, W.F., and Macy, J.M. (eds). New York, USA: Marcell Dekker, pp. 183-215. 
Kashyap, D.R., Botero, L.M., Franck, W.L., Hassett, D.J., and McDermott, T.R. (2006a) Complex regulation of arsenite oxidation in Agrobacterium tumefaciens. J Bacteriol 188: 1081-1088.

Kashyap, D.R., Botero, L.M., Lehr, C., Hasset, D.J., and McDermott, T.R. (2006b) $\mathrm{A} \mathrm{Na}: \mathrm{H}^{+}$antiporter and a molybdate transporter are essential for arsenite oxidation in Agrobacterium tumefaciens. J Bacteriol 188: 15771584.

Koechler, S., Cleiss-Arnold, J., Proux, C., Sismeiro, O., Dillies, M.A., Goulhen-Chollet, F., et al. (2010) Multiple controls affect arsenite oxidase gene expression in Herminiimonas arsenicoxydans. BMC Microbiol 10: 53.

Kraft, T., and Macy, J.M. (1998) Purification and characterization of the respiratory arsenate reductase of Chysiogenes arsenatis. Eur J Biochem 255: 647-653.

Lei, B., Liu, M., Chesney, G.L., and Musser, J.M. (2004) Identification of new candidate vaccine antigens made by Streptococcus pyogenes. J Infect Dis 189: 79-89.

Lett, M.C., Muller, D., Lièvremont, D., Silver, S., and Santini, J. (2011) Unified nomenclature for genes involved in prokaryotic aerobic arsenite oxidation. $J$ Bacteriol doi:10.1128/JB.06391-11.

Lever, J.E. (1972) Purification and properties of a component of histidine transport in Salmonella typhimurium. J Biol Chem 247: 4317-4326.

Lin, Y.F., Yang, J., and Rosen, B.P. (2007) ArsD residues Cys12, Cys13 and Cys18 form an As(III) binding site required for arsenic metallochaperone activity. J Biol Chem 282: 16783-16791.

Liu, Y.G., and Whittier, R.F. (1995) Thermal asymmetric interlaced PCR: automatable amplification and sequencing of insert end fragments from P1 and YAC clones for chromosome walking. Genomics 25: 674-681.

Luecke, H., and Quiocho, F.A. (1990) High specificity of a phosphate transport protein determined by hydrogen bonds. Nature 347: 402-406.

McDermott, T.R., and Kahn, M.L. (1992) Cloning and mutagenesis of the Rhizobium meliloti isocitrate dehydrogenase gene. J Bacteriol 174: 4790-4797.

Macur, R.E., Jackson, C.R., Botero, L.M., McDermott, T.R., and Inskeep, W.P. (2004) Bacterial populations associated with the oxidation and reduction of arsenic in an unsaturated soil. Environ Sci Technol 38: 104-111.

Malasarn, D., Keeffe, J.R., and Newman, D.K. (2007) Characterization of the arsenate respiratory reductase from Shewanella sp. strain ANA-3. J Bacteriol 190: 135-142.

Mascher, T., Helmann, J.D., and Unden, G. (2006) Stimulus perception in bacterial signal-transduction histidine kinases. Microbiol Mol Biol Rev 70: 910-938.

Muller, D., Lievremont, D., Simeonova, D.D., Hubert, J.-C., and Lett, M.-C. (2003) Arsenite oxidase aox genes from a metal-resistant $\beta$-proteobacterium. J Bacteriol 185: 135141.

Nielsen, H., and Krogh, A. (1998) Prediction of signal peptides and signal anchors by a hidden Markov model. In Proceedings of the Sixth International Conference on Intelligent Systems for Molecular Biology. Glasgow, J., Littlejohn, T., Major, F., Lathrop, R., Sankoff, D., and Sensen, C. (eds). Menlo Park, CA, USA: AAAI Press Proceedings, pp. 122-130.
Oremland, R.S., and Stolz, J.F. (2005) Arsenic, microbes and contaminated aquifers. Trends Microbiol 13: 45-49.

Phillips, S.E., and Taylor, M.L. (1976) Oxidation of arsenite to arsenate by Alcaligenes faecalis. Appl Environ Microbiol 32: 392-399.

Pontius, F.W., Brown, K.G., and Chen, C.J. (1994) Health implications of arsenic drinking water. J Am Water Works Assoc 86: 52-63.

Quandt, J., and Hynes, M.F. (1993) Versatile suicide vectors which allow direct selection for gene replacement in gramnegative bacteria. Gene 127: 15-21.

Ruan, X., Bhattacharjee, H., and Rosen, B.P. (2006) Cys-113 and Cys-422 form a high affinity metalloid binding site in the ArsA ATPase. J Biol Chem 281: 9925-9934.

Saltikov, C., and Newman, D.K. (2003) Genetic identification of a respiratory arsenate reductase. Proc Natl Acad Sci USA 100: 10983-10988.

Sambrook, J., Fritsch, E.F., and Maniatis, T. (1989) Molecular Cloning: A Laboratory Manual, 2nd edn. Cold Spring Harbor, NY, USA: Cold Spring Harbor Laboratory Press.

Santini, G.M., and vanden Hoven, R.N. (2004) Molybdenumcontaining arsenite oxidase of the chemolithoautotrophic arsenite oxidizer NT-26. J Bacteriol 186: 1614-1619.

Sardiwal, S., Santini, J.M., Osborne, T.H., and Djordjevic, S. (2010) Characterization of a two-component signal transduction system that controls arsenite oxidation in the chemolithoautotroph NT-26. FEMS Microbiol Lett 313: 20-28.

Shi, W., Wu, J., and Rosen, B.P. (1994) Identification of a putative metal binding site in a new family of metalloregulatory proteins. J Biol Chem 269: 19826-19829.

Somerville, J.E., and Kahn, M.L. (1983) Cloning of the glutamine synthetase I gene from Rhizobium meliloti. J Bacteriol 156: 168-176.

Stolz, J.F., Basu, P., Santini, J.M., and Oremland, R.S. (2006) Arsenic and selenium in microbial metabolism. Annu Rev Microbiol 60: 107-130.

Studier, F.W. (1991) Use of bacteriophage T7 lysozyme to improve an inducible T7 expression system. J Mol Biol 219: 37-44.

Tam, R., and Saier, M.H., Jr (1993) Structural, functional, and evolutionary relationships among extracellular solute-binding receptors of bacteria. Microbiol Rev 57: 320-346.

Thompson, J.D., Gibson, T.J., Plewniak, F., Jeanmougin, F., and Higgins, D.G. (1997) The CLUSTAL_X windows interface: flexible strategies for multiple sequence alignment aided by quality analysis tools. Nucleic Acids Res 25: 4876-4882.

Wanner, B.L. (1996) Phosphorus assimilation and control of the phosphate regulon. In Escherichia coli and Salmonella: Cellular and Molecular Biology, 2nd edn. Neidhardt, F.C., Curtis, R., III, Ingraham, J.L., Lin, E.C.C., Low, K.B., Magasanik, B., et al. (eds). Washington, DC, USA: ASM Press, pp. 1357-1381.

Zargar, K., Hoeft, S., Oremland, R.S., and Saltikov, C. (2010) Identification of a novel arsenite oxidase gene, $\operatorname{arxA}$, in the haloalkaliphilic, arsenite-oxidizing bacterium Alkalilimnicola ehrlichii strain MLHE-1. J Bacteriol 192: 3755-3762. 


\section{Supporting information}

Additional Supporting Information may be found in the online version of this article:

Fig. S1. Clusters of the aio genes in Agrobacterium tumefaciens strain $5 \mathrm{~A}$ and various arsenite-oxidizing bacteria. Representative aio gene clusters are from Xanthobacter autotrophicus Py2 (NC_009720), Roseovarius sp. 217 (NZ_AAMV01000002), Starkeya novella DSM 506 (NC_014217), Rhizobium sp. NT-26 (AY345225), Ochrobactrum tritici strain SCII24 (FJ465505), Alcaligenes faecalis strain NCIB 8687 (AY297781), Rhodoferax ferrireducens T118 (NC_007908), Herminiimonas arsenicoxydans (NC_009138), Thiomonas intermedia K12 (NC_014153), Achromobacter sp. SY8 (EF523515), Alkalilimnicola ehrlichii MLHE-1 (NC_008340), Pseudomonas sp. TS44 (EU311944), Burkholderia multivorans ATCC 17616 (NC_010801), Candidatus Nitrospira defluvii (NC_014355) and Thermus thermophilus HB8 plasmid pTT27 (NC_006462). Homologues were marked as same colour. All aio $\bar{X}$ homologues were indicated as red colour. Note: all aox/aro/aso gene symbols are changed to aio.

Fig. S2. Amino acid alignments of various AioX proteins annotated in different bacteria. The 5A AioX TAT signal peptide is as indicated, with the twin arginines shown as double vertical arrows. The conserved cysteine (aa 108) that was mutated to either an alanine or a serine is also highlighted.

Fig. S3. Diagnostic PCR to verify aioX deletion. A total of $850 \mathrm{bp}$ of nucleotides (grey filled area) is deleted in strain M53. Primer pairs P95/P2218 and P946/P1522 were used to confirm deletion (P95, AGACCCAACACGGAGCG and P2218, CCAGCATTCGTCGCAAGA; P946, GACCTGGAAG TGCTGGACG and P1522, GCTGGCTTTCCCGCTGT).

Fig. S4. Qualitative As(III) oxidation phenotype confirmed by $\mathrm{AgNO}_{3}$ staining. The presence of $\mathrm{As}(\mathrm{V})$ is indicated by dark brown colour associated with the agar as seen when inoculated with WT and M53 carrying pCPP30::aioX. Loss of As(III) oxidation phenotype resulting from an $\Delta$ aio $X$ mutation is also shown in strain M53 ( $\Delta$ aioX mutant) and M53 carrying pCPP30::Cys108Ser. Regions of the agar plate were spot inoculated as indicated. 